\title{
OPTIMIZATION OF ARTIFICIAL NEURAL NETWORKS WITH GENETIC ALGORITHMS FOR BIOMETRIC PATTERN RECOGNITION
}

\author{
Gábor WERNER \\ Óbuda University, Budapest, Hungary \\ wga.bme@gmail.com \\ László HANKA \\ Óbuda University, Budapest, Hungary \\ hanka.laszlo@bgk.uni-obuda.hu
}

\begin{abstract}
The process of pattern recognition in the biometrics is particularly important. The patterns can differ from each other a lot, and even the samples can be significantly different from the templates. The Artificial Neural Networks can be applied as a universal approximator to recognize the patterns with more flexibility. However the topology of the networks determines the processing time and complexity of the hardware of the physical environments. The Genetic Algorithms can be used with success in optimization problems like in this situation, the topology of the neural network is more optimal if we apply the Genetic Algorithms. This study introduce an algorithm in which a tailor made algorithm correcting the topology to enhance the effectiveness of the process.
\end{abstract}

KEYWORDS: biometrics, artificial neural networks, genetic algorithm, pattern recognition, soft-computing, deep-learning

\section{Introduction}

The Artificial Neural Networks (ANN) are widespread used for pattern recognition. The MATLAB (Matrix Laboratory) has a built in toolbox, although we used tailor made algorithm in order to have the most possible variable parameters. Practically the more the parameters we have, the more complicated to find and optimize those. There are some parameters that can be found experimentally, but there are some which are independent from each other and it is difficult to define searching space, thus we are introducing a combination method of two soft-computing method as a deep-learning tool.

According to Hajek (2005), there are a lot of possible structures and models of ANN. In this paper we were focusing to biometrics in which the patterns are quite complex, thus the chosen structure is the Multi Layer Perceptron (MLP) with one input and output layer and two hidden layer (Figure no. 1). 


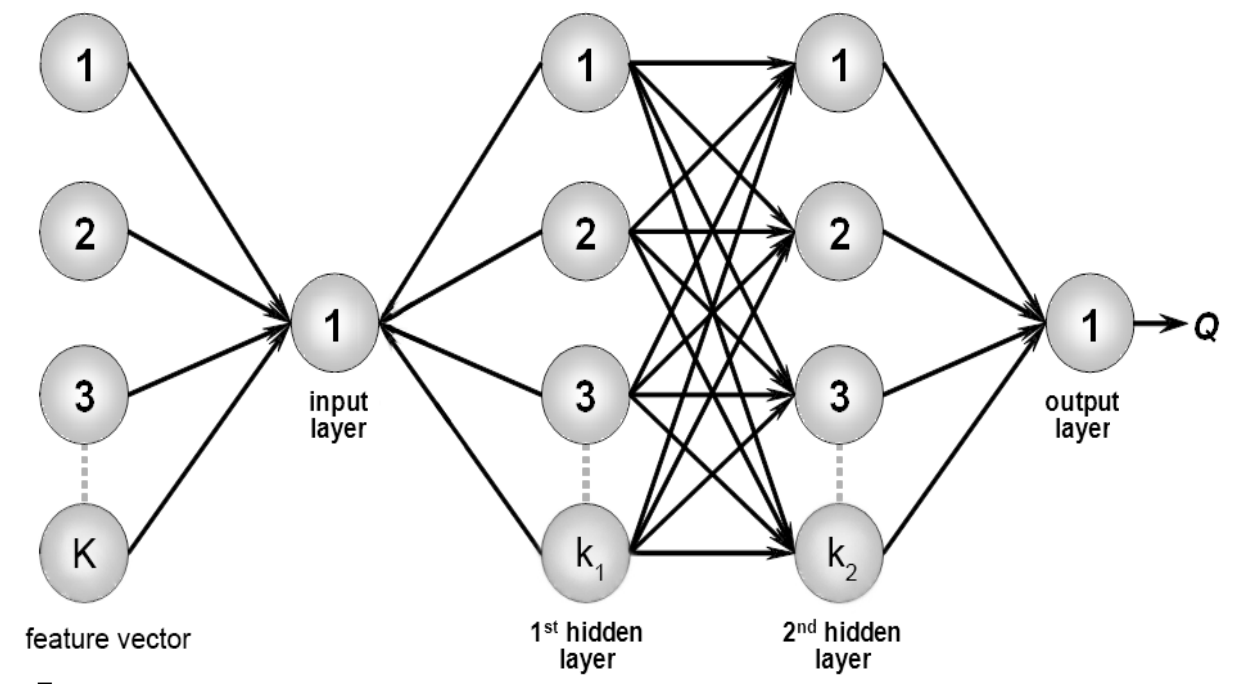

Figure no. 1: Structure of the implemented MLP

In the MLP the size and number of the hidden layers is usually determined to an exact problem. However in our algorithm the number of hidden layer is variable, but fixed to two. The amounts of the neurons in each hidden layers $\left(k_{1}\right.$ and $k_{2}$.) are the researched variables of the Genetic Algorithm (GA), which will be introduced soon. In the ANN it is necessary to give summation and activation functions in each neuron. The character of the activation function has effect on the outcome, the sigmoid function (Figure no. 2) is the most common form, whereas a threshold function assumes the value of 0 or 1 , and it is less sensitively changing close to the extreme values. In this algorithm the activation function form has been chosen fixedly.

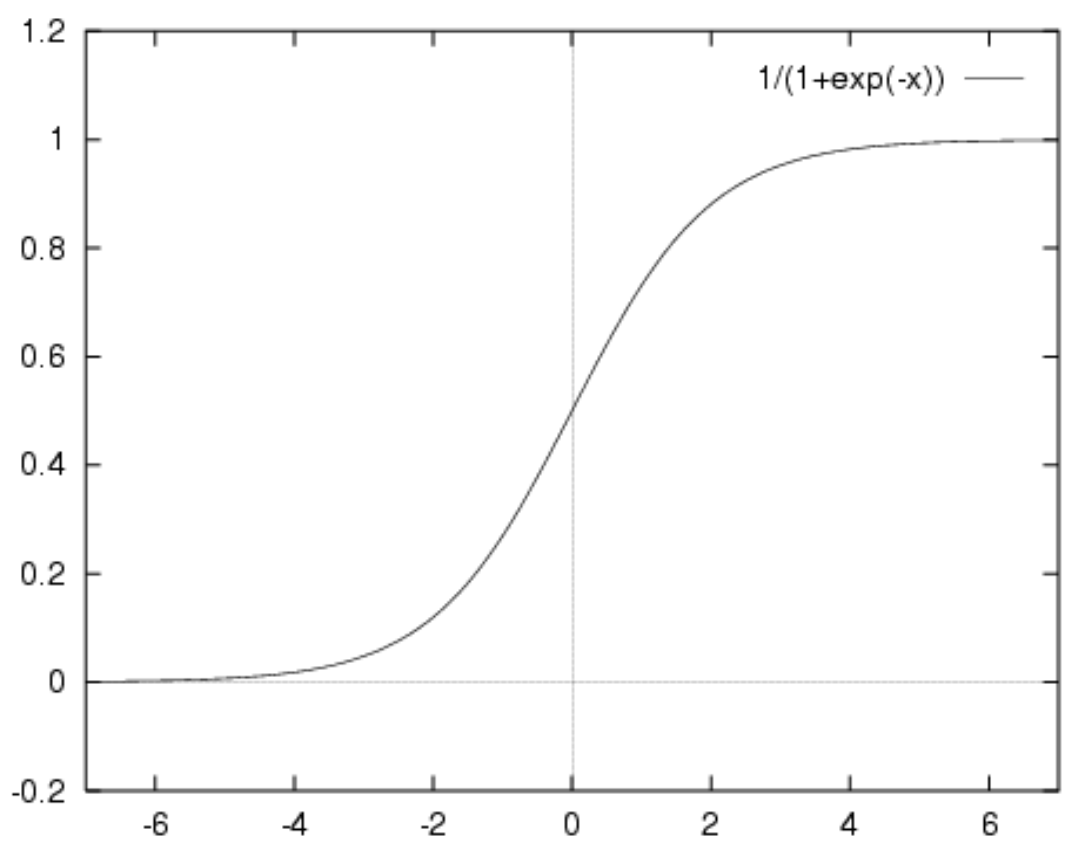

Figure no. 2: Sigmoid activation function

The ANN recently have been used for separation, but as the capacity of network is bigger the complexity of the distinction is more. The advantage of the ANN compared to other classification method is the capability to learn. A neural network is 
learning about its environment through an iterative process of adjustments applied to its synaptic weights and thresholds, similar like in the human brain (Hajek, 2005).

Eventually the ANN is the computational 'copy' of the human brain. The perceptrons are working as the natural neurons, which linked to each other with an organized network. If a link between two neurons is used more likely, then it is getting stronger (weights are higher), so it will have a more effective impact in the decision making (Werner \& Hanka, 2016).

Just like biological brain, this artificial one is able to recognize objects, processes or patterns, but only those ones which have been taught in advance. The level of similarity can be changed in the matching phase or during the training it is even possible to teach more genuine templates to increase the recognition efficiency. The training is necessary but without appropriate test, it is worthless. The test result validates the ANN and its reliability. Generally the usage of the ANN is separated to the following steps (Fazekas, 2013):

1. Network setup;

2. Training phase;

3. Testing phase;

4. Operating phase.

There are some critical setting points in the training phase as well. First of all, it has to be decided how we would like to train. The training here means that, there are an initial weight matrices with random values that symbolize the strength of the links. Actually the starting weight matrices is crucial, because any similarity or regular pattern can lead the learning phase into a local minima, which sabotages the training. Regarding to this phenomenon we used random generator to set the starting weight as it will be shown in the next chapter.

During the training, the algorithm investigates the training patterns, which are mathematically extracted vectors from a biometric samples, or like in our algorithm they are synthesized with a minutiae generator to decrease the environmental failure effects. As all the vectors have been taught then we get result from the output layer. The training is actually a process how the desired and obtained output could getting closer to each other. There are several training methods, we choose a simple and tried one, the Error BackPropagation, accurately Gradient Descent with Momentum Back-Propagation (Werner \& Hanka, 2016).

Depending on the nature of biometric patterns it is useful to use the parameters. One of them is the rate of the learning $(\eta)$, and other is the momentum parameter $\left(\mathrm{m}_{0}\right)$. The rate of the learning $(\eta)$ has to be between 0 and 1, if the value is smaller, then the elements of the weight matrices are changing slower, the trajectory is smoother, but the convergence is slower so. If the eta $(\eta)$ is too close to one, then the convergence is faster, but the algorithm easily become instable, it can be oscillated. To avoid oscillation it is possible to modify the delta rule, which technically means the following:

$\Delta w_{j i}(n)=m_{0} \Delta w_{j i}(n-1)+\eta \delta_{j}(n) y_{i}(n)$

The changing of the elements of the weight matrices $\left(\Delta \mathrm{w}_{\mathrm{ij}}\right)$ is the summation of the change one step before multiply the momentum parameter and the next layer's local gradient. The gradient descent with momentum back propagation make the convergence faster and protect against oscillation (Fazekas, 2013).

Regarding all the parameters that have been mentioned before we can state that the ANN parameters have many possible setting and uncountable variation. Therefore it is necessary to find systematic way to optimize them. In this paper we focused on the topology of the networks, thus the current parameters which will be 
optimized the number of the neurons in the hidden layers and the optimization is provided by most general and metaheuristic tool, the Genetic Algorithm (Melin, Sánchez \& Castillo, 2012).

As other soft computing methods, the GA are also origin from the natural environment. John Holland introduced the GA in 1970, his discovery was inspired by evolution. Since the theory of biological evolution developed by Charles Darwin, it is known the natural creatures are able to adapt to the environmental changes, and also aim to incorporate these alteration into the gene pool (Holland, 1984).

\section{Methods}

The network setup was manually organized, all variable and function have been given in MATLAB regarding the literature and our experiences. In the first phase we made a small program to extract individual identifying marks, which were like minutiae, because the history and mathematical literature of fingerprint recognition is comprehensive. With these artificially constructed fingerprints it is possible to skip the preprocessing and the feature extraction. This means less disturbing factor in the analysis. The red dots are the minutiae that can be analyzed, and blue ones are the middle of the ROIs in the fingerprint reader's scanning windows (position $\mathrm{x}$, position $\mathrm{y}$ ), which symbolize the different templates (Figure no. 3). It is also possible to change the number of the training templates regarding to the flexibility.

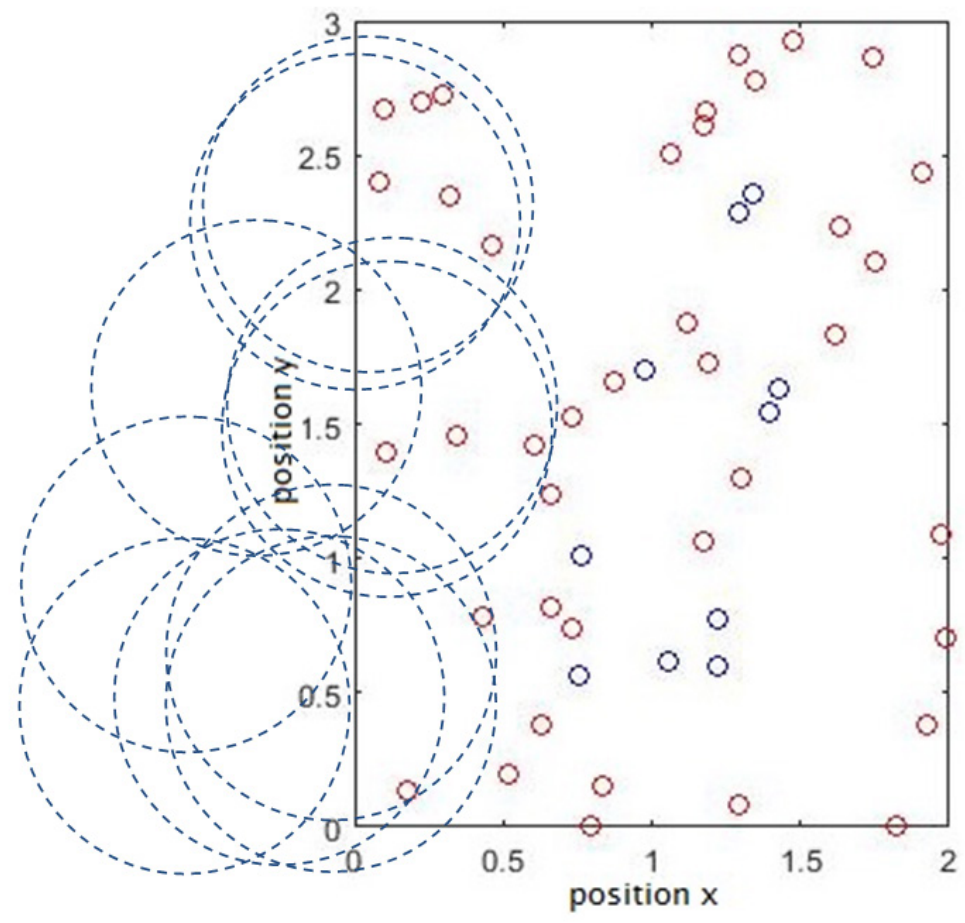

Figure no. 3: Minutiae synthesis in ROI

The Figure no. 3 shows the identifying marks which carry the biometrical information. The number of the minutiae in the ROI has been determined with a variable, thus the number of points to be identified can be adjusted as required. In the minutiae generation we used geometrical features like distances from the two closest neighbors and their angle. These points are endowed with three independent shift- and rotation-resist features (Figure no. 4) which data extracted into the Training database. 


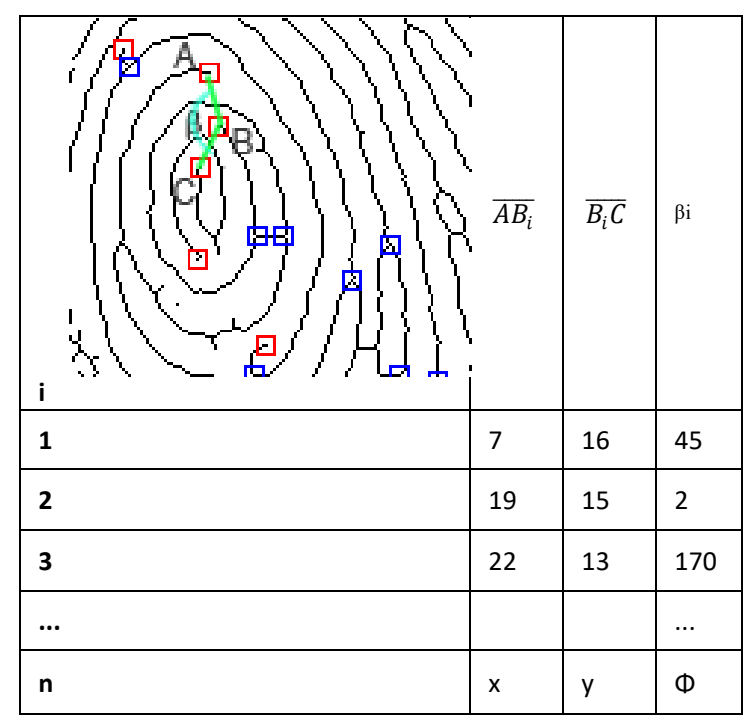

Figure no. 4: Synthesized minutiae with individual identifying marks

The Artificial Neural Networks are highly sensitive to the initial setup of the weight matrices. As we mentioned, if there is any symmetry or initial pattern then the weight matrices will lead to a local minima, and training won't be successful. In a previous study we've investigated that, how it is possible to tuning manually an ANN for increase effectiveness of the training (Werner \& Hanka, 2016).

According to Hajek (2005) the customary practice is to set all the free parameters of the networks to random numbers that are uniformly distributed. Basically the too small initial weights should be avoided, because they are multiplied by $\delta$, (eq. 1.1) and this will cause the error gradient to be too small.

Another inappropriate choice of the initial weights, if they are too large values. This can lead to premature saturation. In this case the output of a neuron approaches the limits of the sigmodial function and only a little change occurs in the weight. The phenomenon corresponds to a saddle point in the error surfaces (Whitley, Starkweather \& Bogart, 1990). Where $\mathrm{x}$ and $\mathrm{y}$ mean the variables of the function that shall be optimized by the back-propagation.

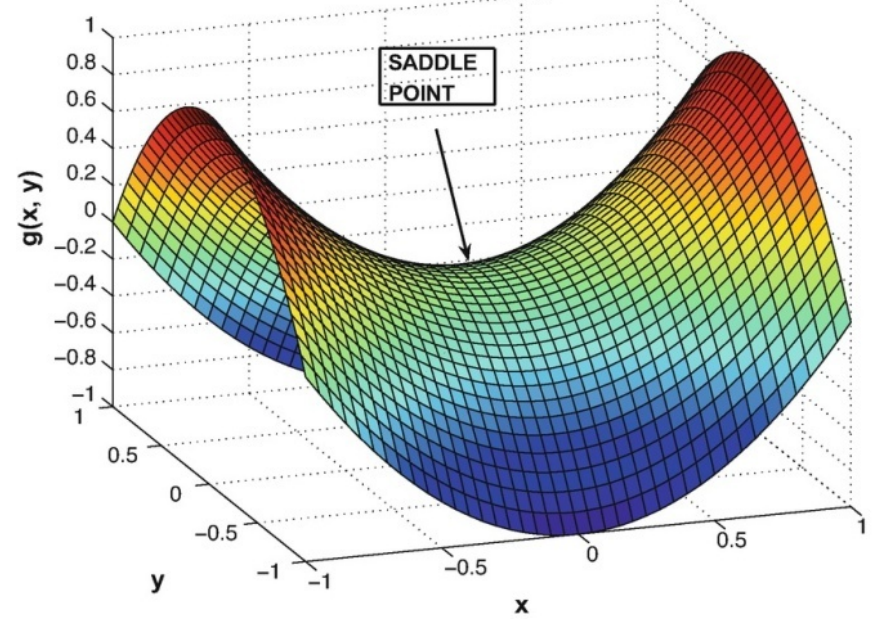

Figure no. 5: Example of saddle point in error surface caused by too big initial weights

(Source: Aggarval, 2018) 
According to our experiments, the manual tuning demonstrated that, the initial distribution of weights in the different layers $\left(\mathrm{w}_{0}=\right.$ input layer; $\mathrm{w}_{1}=$ first hidden layer; $\mathrm{w}_{2}=$ second hidden layer; $\mathrm{w}_{3}=$ output layer) have to be different. Which means that the limits and length of the interval wherefrom the random weight generated have to be chosen different.
To make these differences possible, we set the random generators with different parameters.

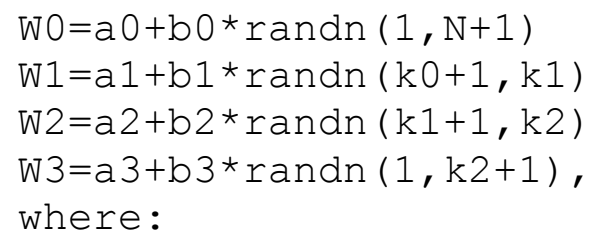

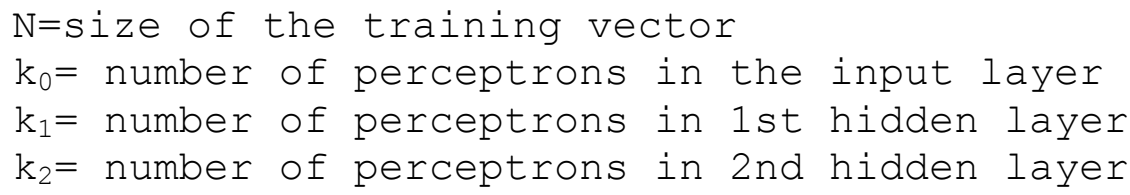

As it has been shown above, there are several variables, that can be changed, and there are numerous parameter which set the efficiency and the characteristic.

In this study we focused on the number of the neurons in the hidden layers, because this is less examined in the scientific literature. The variable of these layers $\left(k_{1}\right.$ and $k_{2}$.) changed from decimal to binary as a chromosome to generate the initial population. The chromosome's length determines the resolution or accuracy of the parameter. The longer the length of a chromosome the wider the possible cart of the variations. All the examined parameters after each other make a string, which contains all chromosomes binary. As in the natural genome, there are several variant of the strings, and the sum of these alleles makes up the population.

According to Darwin's statements, all the populations are trying to survive and multiply their genome, which is actually an endless competition. The better adapting strings have the better chance to survive in a concrete environment or task -, and the degree of the adaptation measured by the fitness value (Holland, 1984).

The biometric devices usually qualified with failure rates, which are able to measure the goodness of a device. Two common rates can be highlighted: the False
Acceptance Rate and the False Rejection Rate. In this study we used these rates, and their combination as fitness value for the Genetic Algorithm.

As the strings are competing which each other, there are three type of modification across the population (selection, crossover, mutation). The bigger the fitness value among the strings the higher the probability of selection into the next generation. The crossover depends on some initial regulation and a random generated probability, it is crossing string fragments into a new whole string. The mutation has an exact small probability that can change a bit in the binary code in each chromosome. The mutation of the probability can be changed if it is necessary (Holland, 1984).

There are several types of selection and crossover methods. We chose the tournament selection type, when for every place in the new population two old strings are competing with each other. They are chosen randomly and the fitter win ever. The crossover is chosen to uniform and the pairing is random, like the position of the crossing point. The probability of the crossover also can be changed depending on the goodness of the GA test's result. The applied structure of the GA is shown in Figure no. 6. 


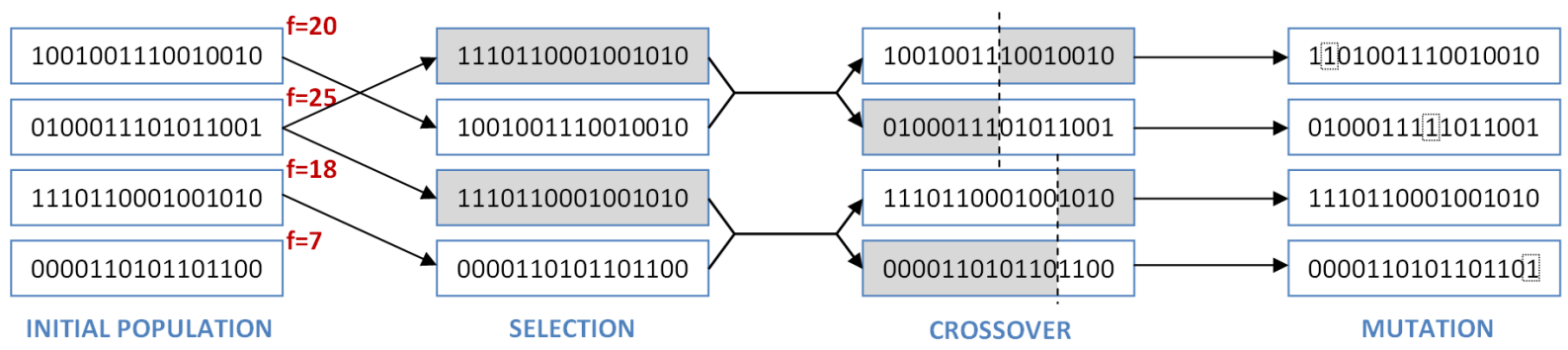

Figure no. 6: Structure of the applied GA

The applied form of the GA for optimization of the ANN still needs some fine tuning. There were some previous decision about the basic architecture like the type of the selection and crossover. Because we didn't want to use the built-in toolbox in MATLAB, we had to considered which GA topology if more effective. We found that the roulette method wouldn't be appropriate for selection, because there were numerous local extreme value among the fitness values.

The crossover type have been changed through the tuning, because we experienced it is favorable to let chance to copy the whole chromosome not only a fragment. The exact point of the brake is determined by a random generator (eq. 1.2).

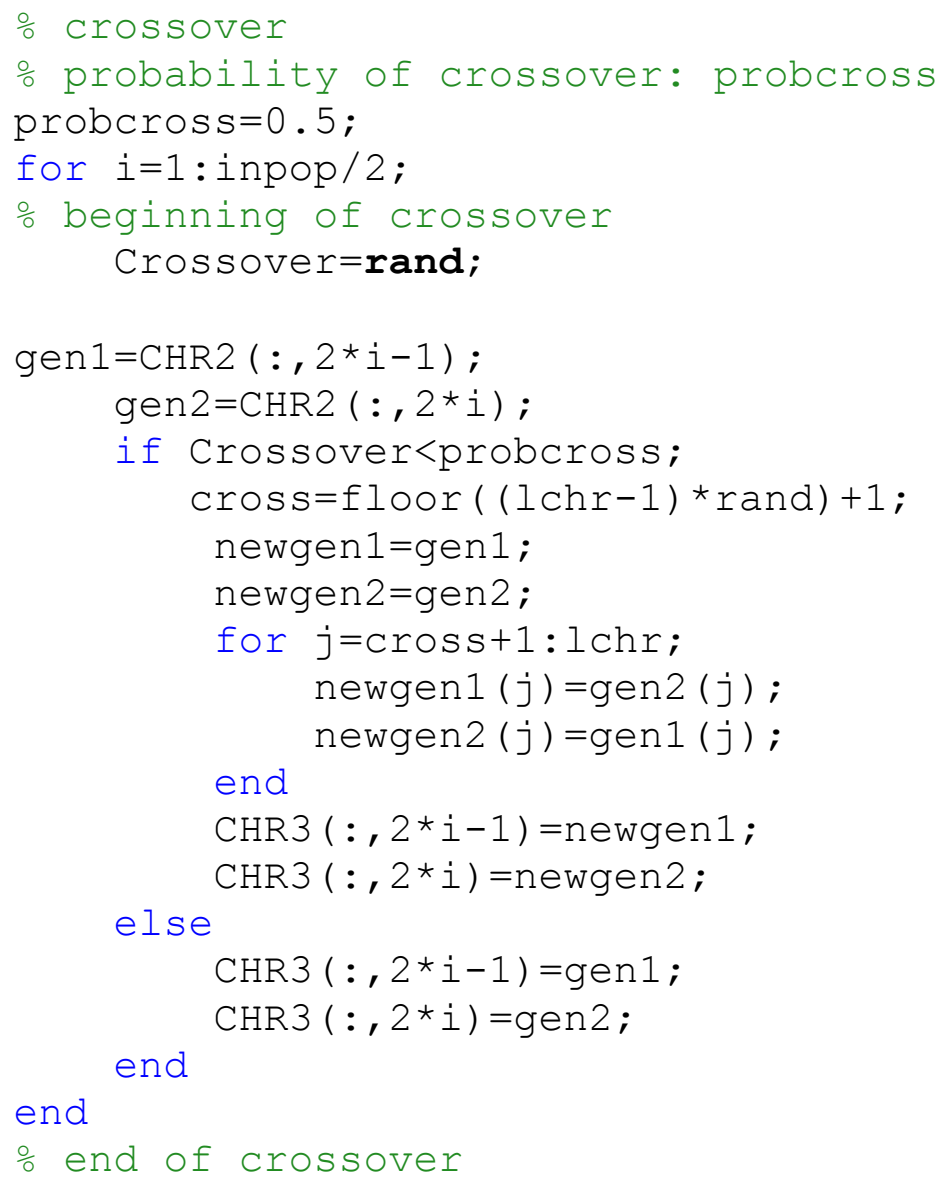


The Figure no. 7 shows the structure of the applied algorithm, which has been encoded in MATLAB. The external cycle is the GA which optimizes the parameters of the ANN. The biometric data generated by a artificial fingerprint algorithm in the way is shown above.

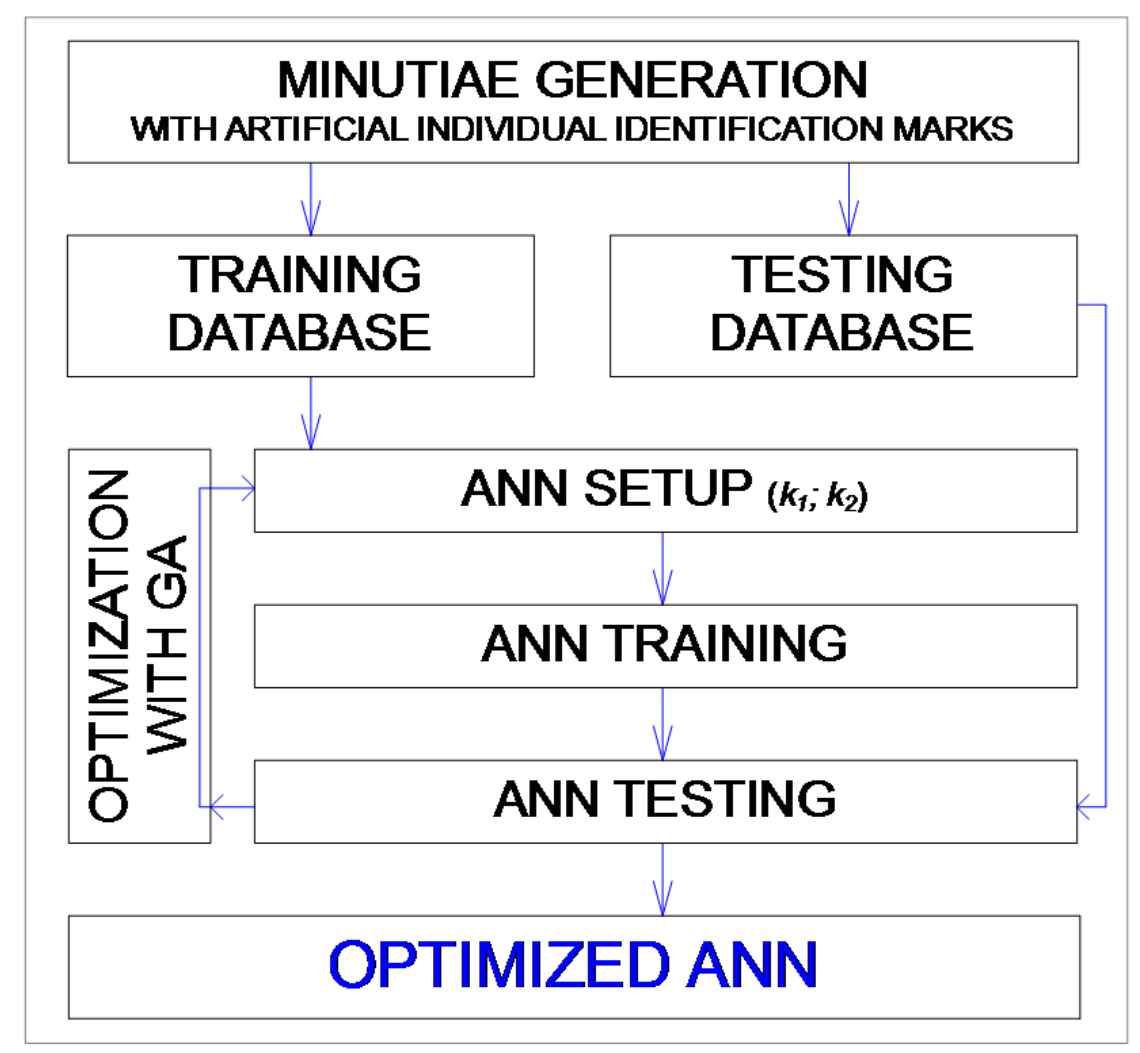

Figure no. 7: Structure of the applied algorithm

\section{Results}

We tried a lot of possible combination of the given adjustable parameters. According to the results it has to be stated the back-propagation is effective if the epsilon $(v)$ and the learning parameter $(\eta)$ are in the same order of magnitude.

In the related studies several types and architecture mentioned of neural networks. Regarding to our experiences, the training is more effective, if the needed number of the minutiae (individual identification marks) is similar or equal with number of the neurons in the input layer. This is easily adjustable if we encode this with a variable that calculates the training data size.
And we can highlight as a result the correlation between the complexity of the investigated database and the topology of the ANN. The complexity in this case refers to the connection between related data sets. The ANN is a good tool to recognize patterns in data sets, and regarding to our experiences if the topology of the ANN is adapting (due to the GA optimization to the complexity, it is possible to spare time or capacity. The Figure no. 8 illustrates the norm of deviance vectors of desired and actual output in the ANN output layer among the actual GA population in each loop. 


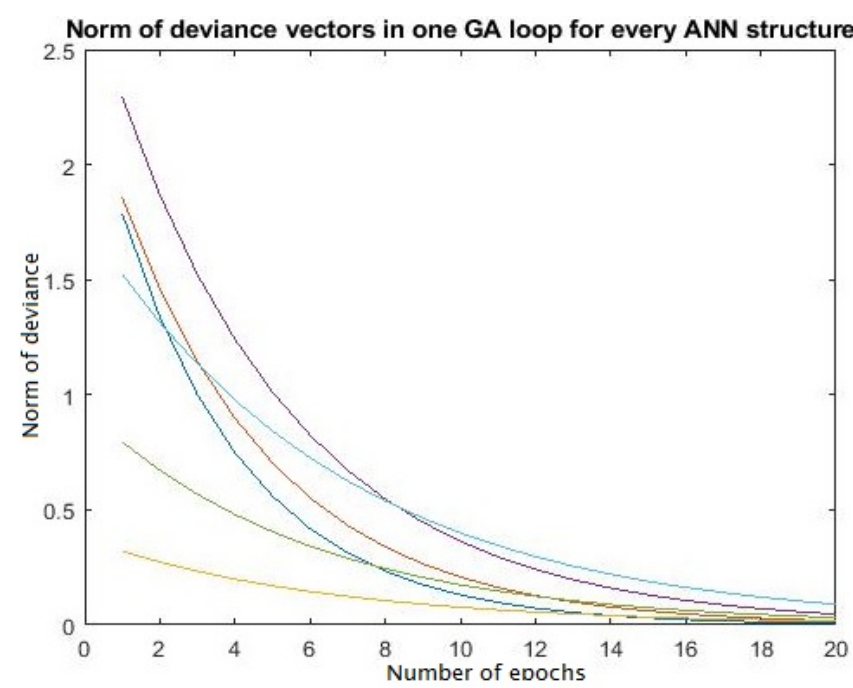

Figure no. 8: Norm of deviance vectors in the GA loops

\section{Conclusions}

Independently from the testing phases with the same settings the algorithm can lead to different result. So the appropriate test is crucial to enhance the comparability. We must emphasize that the structure and the asymmetry of the starting weight matrices have a strong impact on the error back-propagation.
Regarding to the experimental results we can state that the Genetic Algorithm is useful and suitable to find the appropriate ANN topology. The optimization process is mathematically complex, but all biometrical situation are differ from others, and some have special needs which can be easier completed with this technology.

\section{REFERENCES}

Aggarwal, C. C. (2018). Training deep neural networks. Neural Networks and Deep Learning, Springer, 105-167, doi: 10.1007/978-3-319-94463-0_3.

Fazekas, I. (2013). Neural networks. University of Debrecen (Hungary), available at: https://www.tankonyvtar.hu/hu/tartalom/tamop412A/20110103_19_neuralis_halozatok/pr02. html, accessed on 11 June 2019.

Hajek, M. (2005). Neural Networks. University of KwaZulu-Natal, available at: https://www.e-booksdirectory.com/details.php?ebook=10055, accessed on 11 June 2019.

Holland, J. H. (1984). Genetic Algorithms and Adaptation. Adaptive Control of IllDefined Systems. NATO Conference Series (II Systems Science), 16(1), 317-333, doi: 10.1007/978-1-4684-8941-5_21.

Melin, P., Sánchez, D., \& Castillo, O. (2012). Genetic optimization of modular neural networks with fuzzy response integration for human recognition. Information Sciences, Tijuana Institute of Technology, 197(1), 1-19, doi: 10.1016/j.ins.2012.02.027.

Werner, G. A., \& Hanka, L. (2016). Tuning an artificial neural network to increase the efficiency of a fingerprint matching algorithm. 2016 IEEE 14th International Symposium on Applied Machine Intelligence and Informatics (SAMI), 105-109, doi: 10.1109/SAMI.2016.7422990.

Whitley, D., Starkweather, T., \& Bogart, C. (1990). Genetic algorithms and neural networks: optimizing connections and connectivity. Parallel Computing, Computer Science Department, Colorado State University, 14(3), 347-361, doi: 10.1016/0167-8191(90)90086-O. 